\title{
Natural Nanotubes Reinforcing Heterophasic Polypropylene
}

\author{
Suellem Barbosa Cordeiro ${ }^{a}$, Maria de Fátima Vieira Marques ${ }^{a *}$ \\ ${ }^{a}$ Instituto de Macromoléculas Professora Eloisa Mano, Centro de Tecnologia, Universidade \\ Federal do Rio de Janeiro - UFRJ, Rua Horácio Macedo, 2030, Bloco J, CP 68525, \\ CEP 21941-598, Rio de Janeiro, RJ, Brazil
}

Received: April 2, 2014; Revised: February 17, 2015

\begin{abstract}
Nanocomposites of high-impact polypropylene resin and organosilane-functionalized nanotubes in halloysite clay were prepared with the introduction of peroxide to promote selective incorporation of the filler into the polypropylene phase of the matrix, with minor insertion into the EPR elastomeric phase, thus improving the stiffness while preserving the high toughness of the material. Silica composites were also prepared as standard filler. The effect of increasing load content on the morphological and mechanical properties of this material was evaluated by SEM and DMA. The content of the hexaneextracted fraction of these composites after processing was evaluated. Halloysite was well-dispersed in the matrix. Composites with $10 \%$ of filler and $0.5 \% \mathrm{w} / \mathrm{w}$ of dicumyl peroxide had increased stiffness and higher loss factor. An increase of the E' was generated by halloysite addition, but there was a higher decrease in Tan delta. Studies using experimental planning as a tool enabled choosing appropriate amounts of DCP to obtain a high performance composite.
\end{abstract}

Keywords: nanocomposite, polypropylene, halloysite nanotubes, statistics

\section{Introduction}

Heterophasic polypropylene resin is of great industrial interest due to its excellent impact properties. High-impact polypropylene (hiPP) is a type of polypropylene reactor blend formed by an array of isotactic polypropylene (iPP) in which an elastomeric copolymer (EPR) is finely dispersed, with much higher impact resistance at low temperatures than the polypropylene homopolymer, but with lower stiffness, modulus, density and glass transition temperature. It is widely used in injection molding of parts for the automotive industry, rigid packaging for household products, technical parts and coatings of steel pipes ${ }^{1-4}$. However, for broader applications it is necessary to further improve its stiffness/ toughness balance, increasing rigidity without sacrificing impact resistance, although the improvement of one of these properties generally compromises the other.

Relatively few studies have been directly related to strengthening hiPP. However, the literature contains reports of the effect of additives like sodium benzoate (Nabz), talc, bis(3,4-dimethyl-dibenzylidene) sorbitol (DMDBS), and a combination of calcium stearate and pimelic acid (heptanodioic acid). The mechanical properties of heterophasic PP have increased due to the action of these additives as nucleating agents ${ }^{5}$.

Silica is one of the most used fillers in polymer composites ${ }^{6,7}$. Alternatively, halloysite is being used nowadays in diverse polymers ${ }^{8}$. Halloysite is a naturally occurring aluminosilicate nanotube, with a predominantly hollow tubular structure in the submicron range, which is chemically similar to kaolin". It is widely used as filler for paper "sulfite" in plastic and rubber products ${ }^{10}$. Halloysite polymer composites have

*e-mail: fmarques@ima.ufrj.br attracted researchers' interest in recent years due to their unique structures and properties ${ }^{11-16}$. These nanotubes, with molecular formula $\mathrm{Al}_{2} \mathrm{Si}_{2} \mathrm{O}_{5}(\mathrm{OH})_{4} \cdot \mathrm{nH}_{2} \mathrm{O}$, are naturally occurring multi-walled inorganic materials that have similar geometry to carbon nanotubes (CNTs). This aluminosilicate mineral clay can be found in different regions, varying in dimension. The typical size of halloysite is $300-1500 \mathrm{~nm}$ in length, 40-120 $\mathrm{nm}$ for the outer diameter and 15-100 $\mathrm{nm}$ for the inner diameter. Halloysite nanotubes (HNTs) were reported to have remarkable reinforcing effects, high mechanical strength and modulus, enhanced flame retardancy and high dimensional stability, making them excellent for structural and functional applications.

They are considered ideal materials for preparing polymer composites due to four aspects: (i) they are inexpensive because halloysite is abundant; (ii) they are biocompatible ${ }^{17}$; (iii) they are rigid materials with a high aspect ratio; and (ii) compared with other nanoparticles such as fumed silica, montmorillonite and CNTs, they are more easily dispersed in polymer matrixes by shearing. This last advantage in turn has two main reasons: (i) geometrically, the rod-like shape of halloysite is easily dispersed due to the limited intertubular contact area; and (ii) chemically, nanotubes have relatively low hydroxyl density on the outer halloysite surfaces compared with fumed silica and other layered silicates such as montmorillonite ${ }^{14}$.

Therefore, the aggregation induced by the intertubular hydrogen bonding is susceptible to shear force. Study of the morphology of many polymer/halloysite composites has shown single-tube dispersed halloysite in the matrix ${ }^{12,18,19}$. Nanotubes incorporated into polymer composites show promising properties, especially the greatly increased 
mechanical performance. For example, the storage modulus of the epoxy/HNTs hybrid with $12 \mathrm{wt} \%$ HNTs is $58.6 \%$ higher at $50{ }^{\circ} \mathrm{C}$ and $121.7 \%$ higher at $210{ }^{\circ} \mathrm{C}$ than that of the neat epoxy ${ }^{12}$. Polypropylene/HNT and rubber/HNT composites also show about $50 \%$ increased modulus by $30 \mathrm{phr}$ HNTs than those of the neat polymers ${ }^{14,19,20}$.

If nanometer dispersion could be achieved, both mechanical and thermal properties would be further improved or new unexpected features might appear ${ }^{21}$. A great positive effect of additives of nanometric size generally is the very large relative surface areas and strong interactions between the particles and polymer matrix.

Halloysite has superior temperature resistance and due to the easy dispensability of the nanotubes, polymer nanocomposites have relatively uniform distribution in thermoplastics by direct melt blending, especially for polymers with high polarity, although it has been reported that HNTs have been incorporated in several polymers without any surface modification. In fact, especially for low polarity polymers such as polypropylene (PP), improving compatibilization would greatly enhance increase nanocomposite properties.

Due to its highly hydrophobic nature, surface modification of this clay is important ${ }^{22}$. Examples of surface treatment of HNTs are grating maleated polypropylene (PP-MA) onto the nanotubes and adding organic substances with hydrogen-bonding functionalities to form network structures with the hydroxyls and oxygen atoms from Si-O-Si on the HNT surfaces. Another approach is to incorporate some substance on the nanotube surface to promote interfacial reactions between the modified HNTs and the matrix. In this way, grafting of organosilanes could play an important role to improve interfacial wetting with PP. These processes would facilitate the dispersion of HNTs in the matrix and, more importantly, would transfer the stress from the matrix to the filler, improving mechanical performance of the composites, as well as their transparency, attributed to the excellent dispersion of the nanotubular clay.

Mechanical properties, especially the modulus of PP, could be effectively improved with modified HNTs. Due to their hollow structures, HNTs could also be considered with polymers to form low-density nanocomposites, replacing larger quantities of macro or microcompounds. Flexural tensile strength and impact strength can be noticeably improved with the incorporation of HNTs, due to their rodlike morphology and high aspect ratio.

Apart from acting as reinforcing agents, HNTs have also been shown to be effective flame-retardant agents for PP, improving thermal stability and flammability of the polymer. These effects are believed to result from their hollow tubular structure, causing them to act as barriers to heat and mass transport, and the presence of iron in HNTs, making them non-halogen flame-retardant fillers.

In the present work, nanocomposites containing a matrix of heterophasic polypropylene reinforced with halloysite were prepared. Silica was chosen as reference filler because it is already widely used as a reinforcing material. In turn, halloysite is a clay mineral containing tubular nanoparticles and could give excellent mechanical properties due to the formation of PP nanocomposite. The performance of both composites was compared. Moreover, organic peroxide was added to promote selective reinforcement of hiPP.

\section{Material and Methods}

\subsection{Materials}

Halloysite- Premium NZCC- specific surface area $=$ $20 \mathrm{~m}^{2} / \mathrm{g}$ (Imerys Tableware - New Zealand); Silica - 28916 ZEOSIL 175 GR Premium, specific surface area $=174.6 \mathrm{~m}^{2} / \mathrm{g}$ - (Rhodia, Brazil).

The inorganic fillers were pre-heated and subsequently treated with vinyl trimethoxy-silane (VTMS). The product formed was then washed and dried under vacuum.

All the other materials were used as received: dibutyl tin dilaurate (DBTL) - Nuodex (LIOCAT 119) (Miracema); Ethanol P.A. (Vetec Química, Brazil); the antioxidant Irganox 1076 (donated by RioPol); dicumyl peroxide (DCP) $\geq$ 99.0\% -(Sigma-Aldrich); high-impact polypropylene (hiPP) - Grade EP $240 \mathrm{~N}$, pellets, melt flow index of $12 \mathrm{~g} / 10 \mathrm{~min}$. $\left(230{ }^{\circ} \mathrm{C} / 2.16 \mathrm{~kg}\right.$ ) (Quattor S.A.); maleated polypropylene -Polybond 3200 grade (Chemtura Industrial Chemical Ltda., Brazil); vinyl trimethoxy-silane (VTMS) 97\% - (SigmaAldrich); xylene P.A. -(Vetec Química, Brazil).

\subsection{Filler preparation}

To $20 \mathrm{~g}$ of silica, previously sieved through 200 mesh

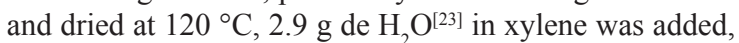
after which the system was kept closed and heated to $80^{\circ} \mathrm{C}$ for $24 \mathrm{~h}$ for the adsorption of water on the surface of the load. Then $20 \mathrm{mmol}$ VTMS per gram of silica was added and about $0.5 \% \mathrm{w} / \mathrm{w}$ DBTL condensation catalyst. This mixture was left under stirring for another 24 hours in an open system for the release of methanol (byproduct of the hydrolysis of VTMS).

The product formed was washed with $50 \mathrm{ml}$ of xylene and then three times with $50 \mathrm{ml}$ of ethanol. When drying, it was characterized by energy-dispersive X-ray spectroscopy (EDX) for elemental analysis, thus determining the content of silicon from the VTMS on the silica surface. The same treatment was performed for halloysite, introducing the same amount of reagent per gram of filler.

The idealized functionalization reaction of the fillers and the reaction of polypropylene on their surface by free radicals (generated by the addition of DCP) are shown in Figures 1 and 2 .

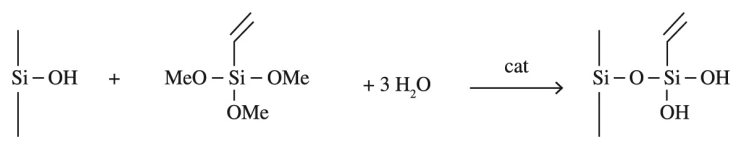

Figure 1. Functionalization reaction of the fillers.

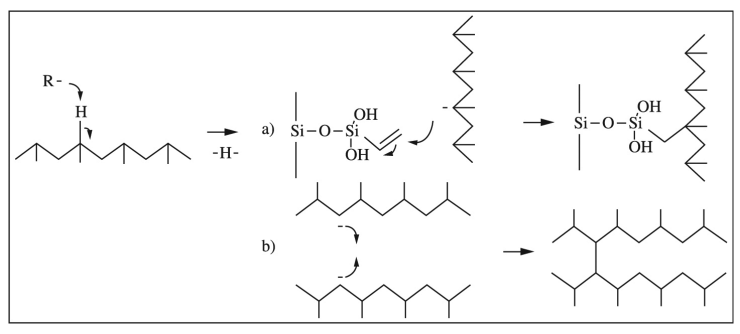

Figure 2. Reaction of grafting the polypropylene chain on the surface of the filler through the vinyl group of fixed VTMS (a). Crosslinking reaction of PP by radicals can also occur (b). 


\subsection{Obtaining composite}

The different fillers were chemically treated to be introduced in the matrix of heterophasic polypropylene (hiPP) with and without the addition of small amounts of DCP, using a Haake Minilab twin-screw mini-extruder in counter-rotating mode for 15 minutes at $40 \mathrm{rpm}$ and $180^{\circ} \mathrm{C}$. In one of the composites (S20d), silica and hiPP were mixed with the use of a compatibilizing agent, polypropylene grafted with maleic anhydride (PP-MA). For comparison, a composite was also prepared with untreated silica. The total mass of sample in the extruder was $5 \mathrm{~g}$. A constant amount $(0.1 \% \mathrm{w} / \mathrm{w})$ of the antioxidant Irganox 1076 was added to the samples.

\subsection{Identification of samples}

Table 1 shows the codes of each sample and the extrusion conditions, employing unmodified fillers or those treated with VTMS, in different amounts. Dicumyl peroxide (DCP) was used because it can allow the reaction of VTMS fixed on the surface of the filler with the polypropylene chains of the continuous phase of hiPP. In turn, the EPR dispersed phase suffers less attack of peroxide due to the presence of less tertiary carbon in its structure. Therefore, it is expected that selective reinforcement could be achieved by this strategy, with the increase of stiffness in the PP continuous phase of hiPP, preserving the EPR phase from the incorporation of fillers, which could cause a decrease in the material's toughness.

During the experimental planning, parameters were estimated having the amount of filler $(10 \%$ to $30 \% \mathrm{w} / \mathrm{w})$ and the concentrations of DCP $(0$ to $0.5 \% \mathrm{w} / \mathrm{w})$ as the variables of interest, and the resulting data were statistically treated. The experiments followed a fractionary factorial plan with two levels ( $2 n$, where $n$ is the number of variables). The factorial plan allows calculating purely empirical equations relating the response variables $\left(\mathrm{E}^{\prime}=\right.$ storage modulus and Tan delta 2 = loss factor of the EPR phase) with the studied

Table 1. Identification of samples.

\begin{tabular}{|c|c|c|c|}
\hline Types of Filler & Sample & $\begin{array}{c}\text { Filler } \\
(\% \mathrm{w} / \mathrm{w})\end{array}$ & $\begin{array}{c}\text { DCP } \\
(\% \text { w/w })\end{array}$ \\
\hline- & hiPP & - & - \\
\hline Untreated Silica & $\mathrm{S} 10^{*}$ & 10 & - \\
\hline \multirow[t]{5}{*}{ Silica/VTMS } & $\mathrm{S} 10$ & 10 & - \\
\hline & $\mathrm{S} 30$ & 30 & - \\
\hline & S10d & 10 & 0.50 \\
\hline & S30d & 30 & 0.50 \\
\hline & $\begin{array}{l}\mathrm{S} 20 \mathrm{~d}_{1} \\
\mathrm{~S} 20 \mathrm{~d}_{2}\end{array}$ & 20 & 0.25 \\
\hline \multirow[t]{5}{*}{ Halloysite/VTMS } & $\mathrm{H} 10$ & 10 & - \\
\hline & $\mathrm{H} 30$ & 30 & - \\
\hline & H10d & 10 & 0.50 \\
\hline & H30d & 30 & 0.50 \\
\hline & $\begin{array}{l}\mathrm{H}_{20 \mathrm{~d}_{1}} \\
\mathrm{H} 20 \mathrm{~d}_{2}\end{array}$ & 20 & 0.25 \\
\hline
\end{tabular}

$\mathrm{S} / \mathrm{H} 10 \mathrm{~d}, \mathrm{~S} / \mathrm{H} 20 \mathrm{~d}$ and $\mathrm{S} / \mathrm{H} 30 \mathrm{~d}=$ samples prepared with increasing amounts filler (silica or halloysite) and DCP; S/H20d = samples containing $20 \%$ filler and $0.25 \%$ DCP were produced in duplicate. S $10 *=$ sample prepared with silica without treatment. parameters. The storage modulus is related to the stiffness of the material while the loss factor can be correlated with the relaxation capacity of the molecules of the EPR disperse phase of the material. Therefore, it can be related to the toughness of the material.

In this work, linear equations were considered. We employed an experimental plan with duplicate at conditions in the central point $(20 \% \mathrm{w} / \mathrm{w}$ of filler and $0.25 \% \mathrm{w} / \mathrm{w}$ of DCP) to estimate the experimental error. To eliminate nonsignificant parameters in the obtained model, those with a significance level above $5 \%(\mathrm{p}>0.05)$ were regarded. The software Statistica version 7.0 from Statsoft was used for the calculations. It should be mentioned that the equations obtained in this study are only valid in the interval studied for the independent variables. According to the estimated parameters, it was possible to predict which effects have statistical significance for the process to obtain improved dynamic-mechanical properties.

\subsection{Characterizations}

The amount of fixed silicon was determined through energy dispersive X-ray spectrometry (EDX) applied to the untreated and VTMS-modified fillers (silica and halloysite). Samples were analyzed in the form of powder, and analysis was carried out under vacuum for 320 seconds. The device used was the EDX-720 (Shimadzu).

The composites were first characterized for the levels of extractable hexane (which is related to the amount of rubbery phase), as well as the virgin polymer. About $1 \mathrm{~g}$ of hiPP and the composites were subjected to extraction under reflux in hexane for 6 hours in a conical extraction system with an HPM6 Basic IKA Labortechnik heating plate, after which the insoluble fraction of the sample was dried in a vacuum oven at $60{ }^{\circ} \mathrm{C}$ to constant weight. The fraction of soluble polymer was determined by the ratio between the mass of the soluble fraction and the initial polymer, deducting the amount of filler added.

Scanning electron microscopy (SEM) was carried out with a SEI Quanta 200 microscope. Micrographs of cryofractured surfaces coated with gold were obtained using electron beam voltage at $5 \mathrm{kV}$, with magnification of 500 to 5,000 times.

Dynamic mechanical analysis (DMA) of the composites was also carried out in a TA Instruments Q800 DMA Analyzer at $10{ }^{\circ} \mathrm{C} / \mathrm{min}$, from $-80^{\circ} \mathrm{C}$ to $140{ }^{\circ} \mathrm{C}$, on single cantilever mode, cycling rate of $1 \mathrm{~Hz}$, and strain of $0.1 \%$. Samples (dimensions $17 \times 12 \times 2 \mathrm{~mm}$ ) were prepared in a hydraulic press at $200{ }^{\circ} \mathrm{C}$ for 10 min under 10000 Psi.

\section{Results and Discussion}

The EDX analysis revealed an increased amount of ${ }_{14} \mathrm{Si}$ on silica and halloysite modified with VTMS, indicating the presence of organo-silane on the surface. By the difference with the original silica, we obtained the silicon content derived from VTMS on the silica surface, which was approximately $0.4 \% \mathrm{w} / \mathrm{w}$, while in halloysite it was $3.4 \% \mathrm{w} / \mathrm{w}$. Probably the higher amount was due to the much higher specific area of halloysite than that of silica ${ }^{24}$, considering the separation of the nanotubes. The reaction of silane on the halloysite surface may also have caused an increase in the space between the nanotubes. 
The hexane-extractable fractions of these composites are presented in Tables 2 and 3.

After extrusion, hiPP presented a higher fraction of hexane-soluble material than the original pellets, showing that in addition to EPR, hexane extracted the fraction of low molecular weight polypropylene, resulting from the molecular weight decrease of the polymer during extrusion due to shear and heat. In the case of the composite samples prepared with DCP containing different amounts of silica/ VTMS, there was generally a lower fraction of hexane extractables, which indicates that the filler reacted with the polypropylene matrix, fixing part of the polymer chains on its surface. The reaction of the polypropylene macroradicals formed under the action of DCP with the vinyl groups fixed on the surface of the filler led to fixing polymer molecules. This should produce good compatibilization of the filler with the polypropylene matrix.

The SEM micrographs of the composites' cryofractured surfaces revealed that sample S20d (obtained introducing $0.25 \% \mathrm{w} / \mathrm{w}$ of DCP during the mixing process and containing $20 \% \mathrm{w} / \mathrm{w}$ of silica) appeared homogeneous (Figure 3c) without plastic deformation, as can be seen in Figure $3 \mathrm{~b}$, in comparison with sample S10* (composite containing untreated silica). Their morphology resembles that of hiPP (Figure 3a)

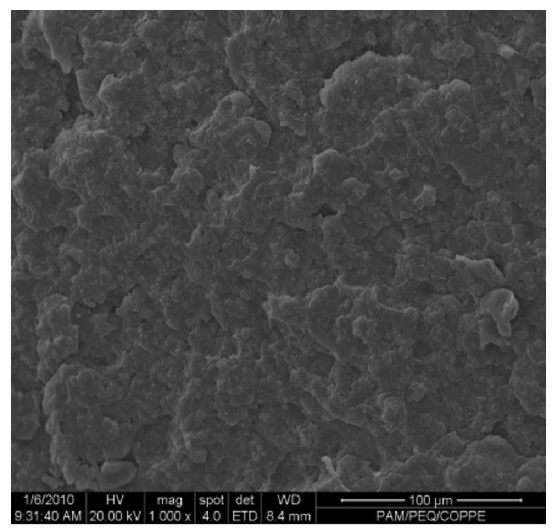

(a) HiPP

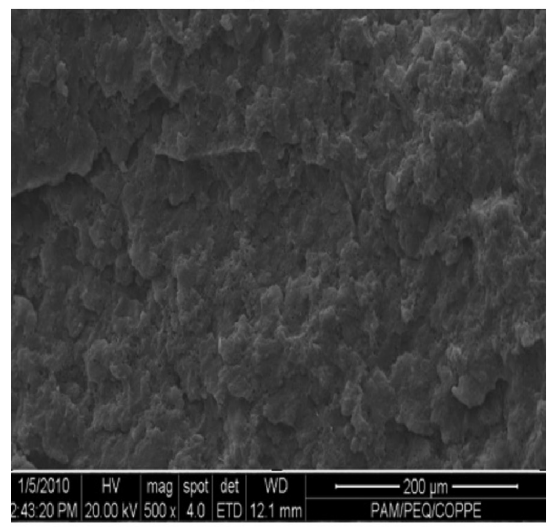

(c) Sample S20d

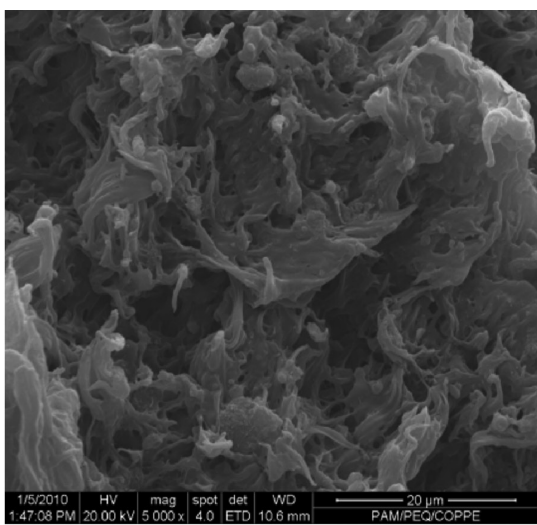

(b) sample $\mathrm{S} 10 *$

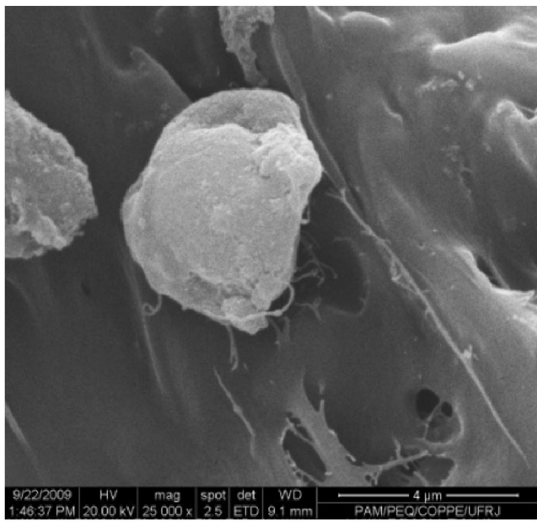

(d) Sample S20d magnified 50x

Figure 3. SEM images: (a) hiPP, (b) Composite S10* (with untreated silica), (c) Composite S20d (20\% w/w silica, with DCP), (d) Composite S20d (50x).

Table 2. Extraction with hexane of the pure hiPP and their composites with silica.

\begin{tabular}{ccccccc}
\hline & \multicolumn{4}{c}{ Samples } \\
\cline { 2 - 7 } Extraction with hexane & \multicolumn{3}{c}{ Without DCP } & \multicolumn{2}{c}{ With DCP } \\
\cline { 2 - 7 } & hiPP (pellets) & hiPP (extruded) & S10 & S30 & S10d & S30d \\
\hline Soluble fraction (\%) & 3.6 & 8.0 & 7.8 & 9.8 & 6.1 & 7.1 \\
\hline
\end{tabular}

Table 3. Extraction with hexane of halloysite composites.

\begin{tabular}{cccccccc}
\hline & \multicolumn{4}{c}{ Samples } \\
\cline { 2 - 8 } Extraction with hexane & \multicolumn{3}{c}{ Without DCP } & \multicolumn{3}{c}{ With DCP } \\
\cline { 2 - 8 } & \multicolumn{2}{c}{ H10 } & H30 & H10d & H30d & H20d $_{1}$ & H20d $_{2}$ \\
\hline Soluble fraction (\%) & 7.1 & 8.3 & 9.3 & 8.3 & 6.7 & 6.5 \\
\hline
\end{tabular}


and indicates the increased compatibility between VTMStreated silica and the matrix, as also shown by Figure $3 \mathrm{~d}$, corresponding to sample 6 with higher magnification.

By analyzing the SEM images of the pure halloysite (Figure 4) and halloysite composites (Figures 4b and 4c), the presence can be noted of isolated nanotubes (white dots), evenly dispersed in the matrix.

The behavior of the hiPP composites was studied by means of dynamic-mechanical analysis in order to determine the storage and loss moduli (Table 4), as well as the glass transition temperatures ( $\mathrm{Tg}$ ) and the damping factor (Tan delta), both in iPP phase (1) and in EPR phase (2).
The results obtained for the composites were compared to those of pure polymer. The results of this study showed that samples S30d and H10d (boldface in the table) have high storage modules (E'), coupled with slight variation in loss factor (Tan delta), which are related to the balance between properties of stiffness/toughness of the hiPP.

After finishing the experiments of the statistical plan for each study, the results of the response variable were placed in the array generated by the Statistica 7.0 program.

Among the results of the storage modulus, E' (Table 4), those with $\mathrm{p}<0.05$ (statistical significance lower than 0.05 , i.e, in the range of $95 \%$ ) are valid and are shown in Figure 5. The

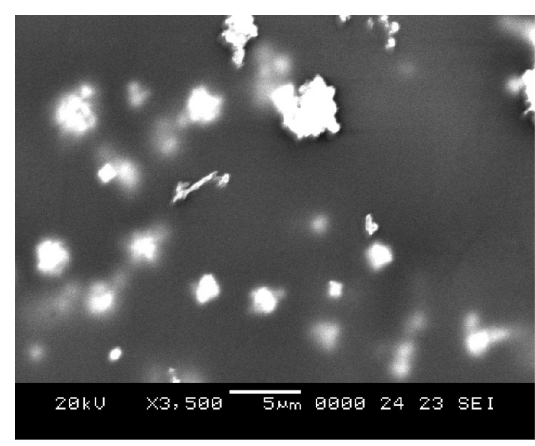

(a) Halloysite

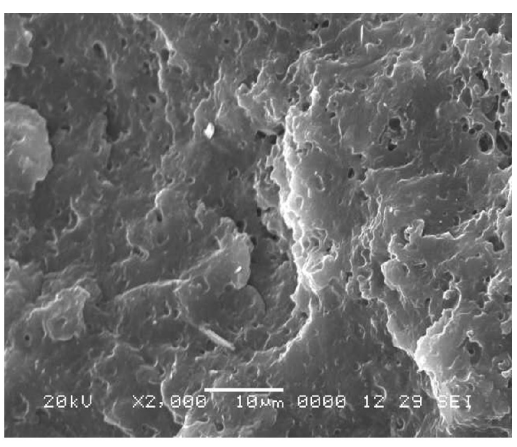

(b) Sample H10

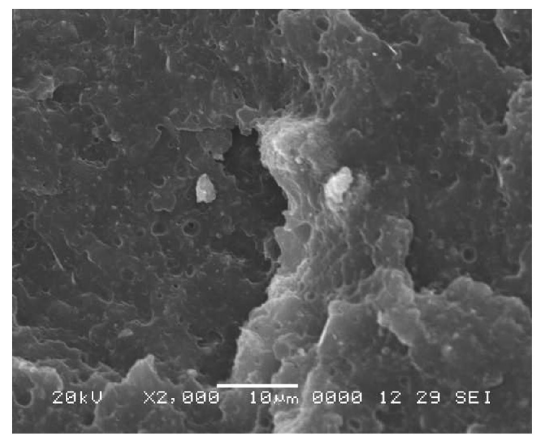

(c) Sample H10d

Figure 4. SEM images: (a) Pure halloysite, (b) Sample H10 (without DCP), (c) Sample H10d (with 0.5 w/w DCP), both containing $10 \%$ w/w halloysite.

Table 4. Results obtained by DMA of the composites and hiPP.

\begin{tabular}{|c|c|c|c|c|c|c|}
\hline Samples & $\begin{array}{l}\mathbf{E}^{\prime} \\
\left(\mathbf{M P a}{ }^{\circ} \mathrm{C}\right.\end{array}$ & $\begin{array}{l}\mathbf{E}{ }^{2{ }^{\circ} \mathrm{C}} \\
(\mathrm{MPa})\end{array}$ & $\begin{array}{c}\operatorname{Tg}_{1}\left(E^{\prime \prime}\right) \\
\left({ }^{\circ} \mathbf{C}\right)\end{array}$ & $\begin{array}{c}\operatorname{Tg}_{2}\left(E^{\prime \prime}\right) \\
\left({ }^{\circ} \mathbf{C}\right)\end{array}$ & $\begin{array}{c}\text { Tan delta1 } \\
\text { Max }\end{array}$ & $\begin{array}{c}\text { Tan delta } 2 \\
\text { Max }\end{array}$ \\
\hline hiPP & 806 & 38.1 & 2.4 & -45.1 & 0.05226 & 0.06515 \\
\hline $\mathrm{S} 10$ & 668 & 33.7 & 0.5 & -38.8 & 0.05708 & 0.04465 \\
\hline $\mathrm{S} 30$ & 831 & 39.2 & 4.0 & -40.1 & 0.05161 & 0.05705 \\
\hline S10d & 1001 & 52.8 & 1.5 & -45.1 & 0.05412 & 0.05342 \\
\hline S30d & 955 & 45.9 & 2.1 & -44.3 & 0.05100 & 0.06324 \\
\hline $\mathrm{S}^{2} 0 \mathrm{~d}_{1}$ & 783 & 47.0 & 1.6 & -45.0 & 0.06268 & 0.06721 \\
\hline $\mathrm{S} 20 \mathrm{~d}_{2}$ & 775 & 46.6 & 1.1 & -45.5 & 0.06266 & 0.06775 \\
\hline $\mathrm{H} 10$ & 646 & 36.5 & 2.5 & -45.5 & 0.06033 & 0.06141 \\
\hline $\mathrm{H} 30$ & 934 & 54.4 & 2.7 & -44.9 & 0.06240 & 0.05372 \\
\hline H10d & 980 & 54.0 & 2.9 & -41.9 & 0.06091 & 0.06234 \\
\hline $\mathrm{H} 30 \mathrm{~d}$ & 1001 & 62.6 & 3.6 & -43.3 & 0.06742 & 0.05520 \\
\hline $\mathrm{H} 20 \mathrm{~d}_{1}$ & 1918 & 121.1 & 2.3 & -45.6 & 0.06055 & 0.05204 \\
\hline $\mathrm{H} 20 \mathrm{~d}_{2}$ & 1851 & 109.0 & 2.3 & -44.5 & 0.05887 & 0.05059 \\
\hline
\end{tabular}

$E^{\prime}=$ storage modulus; $E "=$ loss modulus; $\mathrm{Tg}_{1}(\mathrm{E} ")=$ glass transition temperature of the PP continuous phase of hiPP obtained through E"; $\mathrm{Tg},(\mathrm{E} ")=\mathrm{Tg}$ of the EPR dispersed phase of hiPP obtained through E"; Tan delta 1 and Tan delta $2=$ loss factor of PP continuous phase and EPR dispersed phase of hiPP, respectively. $\mathrm{S} 20 \mathrm{~d}_{1}$ and $\mathrm{S} 20 \mathrm{~d}_{2}$ are duplicate, as well as $\mathrm{H} 20 \mathrm{~d}_{1}$ and $\mathrm{H}_{2} 0 \mathrm{~d}_{2}$. 

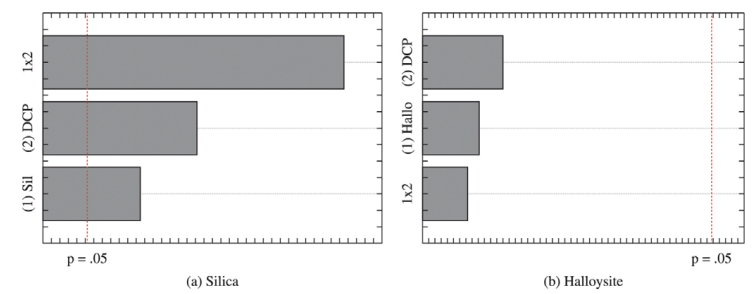

Figure 5. Pareto diagrams: Effect of varying concentrations of DCP and amount of filler on the response variable storage modulus. (a) Silica, and (b) Halloysite.

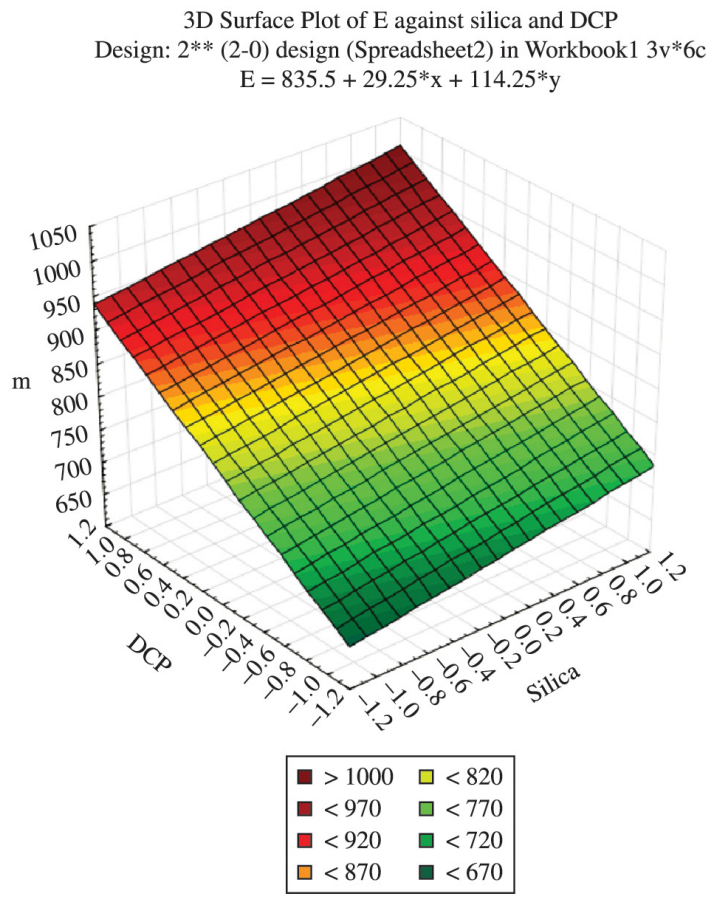

Figure 6. Surface Graph of the composite with silica in the experimental design for $\mathrm{E}^{\prime}$. Where $\mathrm{X}=$ silica content, $\mathrm{Y}=\mathrm{DCP}$ content and $\mathrm{E}=$ modulus.

factor analyzed is statistically significant when the value of the statistical significance $p$ is below the required significance.

The dotted line expresses the statistical significance used $(0.05)$ and represents the minimum value that the effect must have to be considered a significant variable. The parameters whose effects go beyond this line are statistically significant. Therefore, only the parameters of samples containing silica presented statistical significance. On the other hand, for the samples containing halloysite, the parameters in the studied range did not have statistical significance. This means no linear relation between the storage modulus and loss factor was obtained.

In the experimental design for the samples containing silica, a linear equation for the model (Equation 1) was obtained, as follows:

$$
E^{\prime}=(835.5)+(29.25) \times S i l+(-104.75) \times D C P+(114.25) \times S i l \times D C P
$$

where:

$\mathrm{E}^{\mathrm{c}}=$ storage modulus;

Sil $=$ silica content $(\% \mathrm{w} / \mathrm{w})$;

$\mathrm{DCP}=\mathrm{DCP}$ content $(\% \mathrm{w} / \mathrm{w})$.

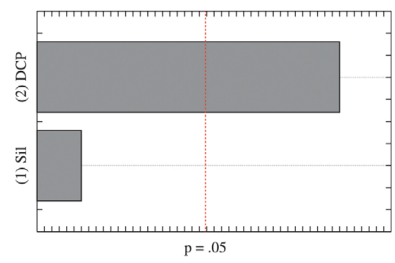

Figure 7. Pareto diagrams: Effect of variables: the amount of filler and DCP concentrations on the response variable Tan delta 2 in silica.

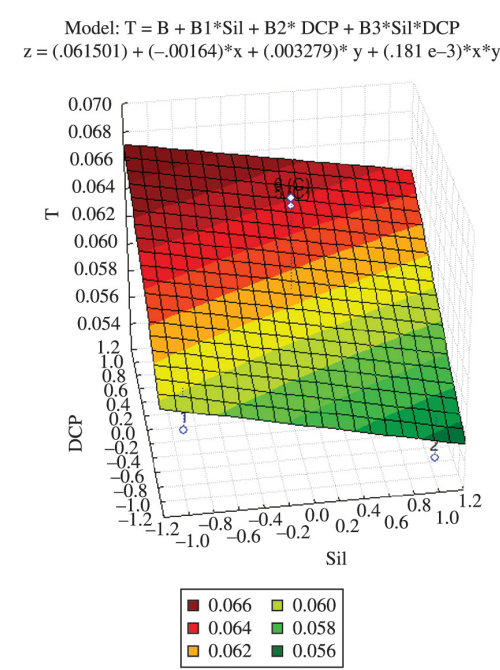

Figure 8. Chart of composites with silica surface in the experimental design for Tan delta 2.

A correlation coefficient of 0.99572 was obtained. Thus, the surface graph obtained is shown in Figure 6. This graph shows, for example, that a material with high values of E' (dark areas in the graph) should be used at lower concentrations of DCP and an increased amount of silica in the range used in this study.

When considering the value of Tan delta 1 (loss factor of the EPR dispersed phase) as the dependent variable, the composites containing silica obtained statistical significance. Figure 7 presents the Pareto diagram showing the effect of the independent variables (amount of silica and concentration of DCP) on the dependent variable (Tan delta 2) in the samples containing silica. It can be observed that the DCP content variable was significant.

For the samples containing silica, the linear equation model (Equation 2) for Tan delta 2 was as follows:

$$
\begin{aligned}
& \tan \delta=(0.061501)+(-0.00164) \times S i l+(0.003279) \times \\
& D C P+\left(1.81 \times 10^{-4}\right) \times \text { Sil } \times D C P
\end{aligned}
$$

where:

$\operatorname{Tan} \delta=$ loss factor of the dispersed phase (Tan delta 2);

Sil $=$ silica content $(\% \mathrm{w} / \mathrm{w})$;

$\mathrm{DCP}=\mathrm{DCP}$ content $(\% \mathrm{w} / \mathrm{w})$.

A correlation coefficient of 0.78810 was obtained. The surface graph obtained is shown in Figure 8. 


\section{Conclusion}

For the fillers studied, the treatment with VTMS was effective since silicon was fixed, as confirmed by EDX. Sample S20d had the highest compatibility among the composites containing silica, which was observed by SEM, showing the polymer coating on the surface of the filler. In halloysite composites, the nanotubes were observed isolated and dispersed uniformly in the PP matrix. Among the samples containing halloysite, those with DCP appeared to have more compatibilization in the composites. In the composites containing $10 \% \mathrm{w} / \mathrm{w}$ using the fillers studied,

\section{References}

1. Chum PS and Swogger KW. Olefin polymer technologiesHistory and recent progress at The Dow Chemical Company. Progress in Polymer Science. 2008; 33(8):797-819. http:// dx.doi.org/10.1016/j.progpolymsci.2008.05.003.

2. Lotti C, Correa CA and Canevarolo SV. Mechanical and morphological characterization of polypropylene toughened with olefinic elastomer. Materials Research. 2000; 3(2):37-44. http://dx.doi.org/10.1590/S1516-14392000000200007.

3. Braskem. São Paulo. Available from: $<w w w . b r a s k e m . c o m . b r>$.

4. Urdampilleta I, González A, Iruin JJ, De La Cal JC and Asua JM. Morphology of impact polypropylene particles. Macromolecules. 2005; 38(7):2795-2801. http://dx.doi.org/10.1021/ma047413v.

5. Trongtorsak K, Supaphol P and Tantayanon S. Effect of calcium stearate and pimelic acid addition on mechanical properties of heterophasic isotactic polypropylene/ethylene-propylene rubber blend. Polymer Testing. 2004; 23(5):533-539. http:// dx.doi.org/10.1016/j.polymertesting.2003.11.006.

6. Foo E, Jaafar M, Aziz A and Sim LC. Properties of spin coated epoxy/silica thin film composites: Effect of nano- and micron-size fillers. Composites. Part A, Applied Science and Manufacturing. 2011; 42(10):1432-1437. http://dx.doi. org/10.1016/j.compositesa.2011.06.007.

7. Chen J, Yang MS and Zhang SM. Immobilization of antioxidant on nanosilica and the aging resistance behavior in polypropylene. Composites. Part A, Applied Science and Manufacturing. 2011; 42(5):471-477. http://dx.doi.org/10.1016/j.compositesa.2011.01.006.

8. Du M, Guo B and Jia D. Newly emerging applications of halloysite nanotubes: a review. Polymer International. 2010; 59:574-582.

9. Oliveira MTG, Furtado SMA, Formoso MLL, Eggleton RA and Dani N. Coexistence of halloysite and kaolinite: a study on the genesis of kaolin clays of Campo Alegre Basin, Santa Catarine State, Brazil. Anais da Academia Brasileira de Ciências. 2007; 79(4):665-681. http://dx.doi.org/10.1590/ S0001-37652007000400008.

10. Ece ÖI and Schroeder PA. Clay mineralogy and chemistry of Halloysite and Alunite deposits in the Turplu area, Balikesir. Clays and Clay Minerals. 2007; 55(1):18-35. http://dx.doi. org/10.1346/CCMN.2007.0550102.

11. Ye Y, Chen H, Wu J and Ye L. High impact strength epoxy nanocompósitos with natural nanotubes. Polymer. 2007; 48(21):6426-6433. http://dx.doi.org/10.1016/j.polymer.2007.08.035.

12. Ning N, Yin Q, Luo F, Zhang Q, Du R and Fu Q. Crystallization behavior and mechanical properties of polypropylene/halloysite composites. Polymer. 2007; 48(25):7374-7384. http://dx.doi. org/10.1016/j.polymer.2007.10.005.

13. Liu M, Guo B, Du M, Cai X and Jia D. Properties of Halloysite nanotube- epoxy resin hybrids and the interfacial reactions in the systems. Nanotechnology. 2007; 18(45):455703-455711. http://dx.doi.org/10.1088/0957-4484/18/45/455703. those with the addition of DCP presented superior mechanical performance, with improved balance of stiffness/toughness of the hiPP. Through the experimental planning it was possible to determine the appropriate quantities of DCP and silica to produce composites with high values of storage modulus and loss factor (Tan delta 2).

\section{Acknowledgements}

We thank the National Research Council (CNPq) and the Office to Improve University Personnel (CAPES), Brazil, for supporting this work.

14. Liu, M, Guo, B, Du, M, Jia, D. Drying induced aggregation of Halloysite nanotubes in polyvinyl alcohol/halloysite nanotubes solution and its effect on properties of composite film. Applied Physics A. 2007; 88(2):391-395.

15. Levis SR and Deasy PB. Characterisation of halloysite for use as a microtubular drug delivery system. International Journal of Pharmaceutics. 2002; 243(1-2):125-134. http://dx.doi. org/10.1016/S0378-5173(02)00274-0. PMid:12176301

16. Liu M, Guo B, Zou Q, Du M and Jia D. Interactions between halloysite nanotubes and 2,5-bis(2-benzoxazolyl) thiophene and their effects on reinforcement of polypropylene/halloysite nanocomposites. Nanotechnology. 2008; 19(20):205709. 205718. http://dx.doi.org/10.1088/0957-4484/19/20/205709. PMid:21825752

17. Hedicke-Höchstötter KH, Lim GT and Altstädt V. Novel polyamide nanocomposites based on silicate nanotubos of the mineral halloysite. Composites Science and Technology. 2009; 69(34):330-334. http://dx.doi.org/10.1016/j.compscitech.2008.10.011.

18. Lvov YM, Shchukin DG, Möhwald H and Price RR. Halloysite clay nanotubes for controlled release of protective agents. ACS Nano. 2008; 2(5):814-820. http://dx.doi.org/10.1021/nn800259q. PMid:19206476

19. Liu M, Guo B, Du M, Chen F and Jia D. Halloysite nanotubes as a novel $\beta$-nucleating agent for isotactic polypropylene. Polymer. 2009; 50(13):3022-3030. http://dx.doi.org/10.1016/j. polymer.2009.04.052.

20. Wang C and Liu CR. Transcrystallization of polypropylene composites: nucleating ability of fibres. Polymer. 1999; 40(2):289298. http://dx.doi.org/10.1016/S0032-3861(98)00240-7.

21. Ismail H, Pasbakhsh P, Fauzi MNA and Abu Bakar A. Morphological, thermal and tensile properties of halloysite nanotubes filled ethylene propylene diene monomer (EPDM) nanocomposites. Polymer Testing. 2008; 27(7):841-850. http:// dx.doi.org/10.1016/j.polymertesting.2008.06.007.

22. Yuan P, Southon PD, Liu Z, Green MER, Hook JM, Antill SJ, et al. Functionalization of halloysite clay nanotubes by grafting with gama aminopropyltriethozylane. The Journal of Physical Chemistry C. 2008; 112(40):15742-15751. http:// dx.doi.org/10.1021/jp805657t.

23. Yoshida W, Castro RP, Jou JD and Cohen Y. Multilayer Alkoxysilane Silylation of Oxide Surfaces. Langmuir. 2001; 17(19):5882-5888. http://dx.doi.org/10.1021/la001780s.

24. Rooj S, Das A, Thakur V, Mahaling RN, Bhowmick AK and Heinrich G. Preparation and properties of natural nanocomposites based on natural rubber and naturally occurring halloysite nanotubos. Materials \& Design. 2010; 31(4):2151-2156. http:// dx.doi.org/10.1016/j.matdes.2009.11.009. 